\title{
Tsunami-induced magnetic fields detected at Chichijima Island before the arrival of the 2011 Tohoku earthquake tsunami
}

\author{
Hidee Tatehata ${ }^{1 *}$, Hiroshi Ichihara ${ }^{2,3}$ and Yozo Hamano ${ }^{4}$
}

\begin{abstract}
Magnetic field disturbances associated with the tsunami caused by the 2011 Tohoku earthquake were observed at Chichijima Island, $1200 \mathrm{~km}$ south of the epicenter. The vertical component of the magnetic field showed a periodic signal at approximately 20 min before the tsunami arrived. This study investigated the mechanism of the magnetic field signal using simulation studies. First, we derived a tsunami source model that explained the tide gauge records and sea-level changes at Chichijima Island. Using this model, we then computed the electric current induced by the tsunami and the resulting secondary magnetic field. The computed changes in sea level and magnetic field are consistent with their respective observed waveforms, including the timing of the magnetic field signals. In our interpretation, the tsunami flow induced an electric current along the tsunami wave front, which in turn generated a secondary induced magnetic field ahead of the tsunami wave. Hence, magnetic variations preceding the tsunami were observed at Chichijima Island. This suggests that imminent arrivals of tsunamis can be detected by observations of the magnetic field.
\end{abstract}

\section{Findings} Introduction

The oceanic dynamo effect was anticipated theoretically by Michael Faraday (Faraday 1832) and has been studied in further detail during recent decades (e.g., Sanford 1971; Tyler 2005; Tyler et al. 2003). With the advancement of observational devices, tsunami-induced dynamo effects have recently been observed on the seafloor (Toh et al. 2011; Ichihara et al. 2013; Sugioka et al. 2014; Zhang et al. 2014a). A few instances of these effects have been documented on land. One was a magnetic signal associated with the 27 February 2010 earthquake (M8.8) off the coast of Chile, which was observed at Easter Island, $3500 \mathrm{~km}$ from the epicenter (Manoj 2011). A magnetic signal associated with the tsunami caused by the 11 March 2011 Tohoku earthquake (M9.0) was observed in Chichijima Island, from which a clear signal of the oceanic dynamo effect was reported by Zhang et al. (2014b). They reproduced the observed magnetic field but did not perform a comparison between the tsunami and the magnetic field.

In this paper, we report on tsunami-induced magnetic field variations that were observed at Chichijima Island at approximately $20 \mathrm{~min}$ before the tide gauge there recorded the arrival of the tsunami.

The aims of this study were to confirm that the oceanic dynamo effect caused the magnetic field variations and to understand the mechanism that allowed the detection of the magnetic field variations before the sea-level change. In this paper, we first present and compare the sea-level changes and magnetic field variations after the 2011 Tohoku earthquake. Next, we report on our numerical simulations of tsunamis and induced magnetic fields at Chichijima Island to explain the corresponding observations. Finally, we discuss the mechanism by which the tsunami-induced magnetic field can signal a tsunami before the occurrence of changes in sea level.

\footnotetext{
* Correspondence: tatehata@met.kishou.go.jp

${ }^{1}$ Kakioka Magnetic Observatory, Japan Meteorological Agency, 595 Kakioka,

Ishioka-shi, Ibaraki-ken 315-0116, Japan

Full list of author information is available at the end of the article
}

\section{国 Springer}

(C) 2015 Tatehata et al. Open Access This article is distributed under the terms of the Creative Commons Attribution 4.0 International License (http://creativecommons.org/licenses/by/4.0/), which permits unrestricted use, distribution, and reproduction in any medium, provided you give appropriate credit to the original author(s) and the source, provide a link to the Creative Commons license, and indicate if changes were made. 


\section{Observations at Chichijima Island \\ Tide gauge}

Chichijima Island is situated on the Izu-Bonin-Mariana Ridge, about $1200 \mathrm{~km}$ south-southwest of the 2011 Tohoku tsunami source (Fig. 1). The tide gauge station, operated by the Japan Meteorological Agency (JMA), is on the northern side of Futami harbor and has an ultrasonic distance meter that measures sea-level changes at intervals of $15 \mathrm{~s}$.

\section{Magnetic observations}

Magnetic field variations were observed at the Chichijima geomagnetic observation station (CBI), located $155 \mathrm{~m}$ above sea level on a hill approximately $1 \mathrm{~km}$ from the tide gauge station (Fig. 1). To compare the record of magnetic field variations with records from another site, we also used records from the Kanoya geomagnetic station (KNY) at the southern end of Kyushu Island, about $1000 \mathrm{~km}$ from Chichijima Island. Both observation stations are operated by the JMA and are equipped with fluxgate magnetometers with a resolution of $0.1 \mathrm{nT}$. Continuous time series of three magnetic components at an interval of $1 \mathrm{~s}$ are available from both stations (Fig. 2). We applied a band-pass digital filter (transmission period of 3-60 min) to remove diurnal variations and short-period instrumental noise.

Figure 2 shows the horizontal component $H$ and vertical component $Z$ of the filtered geomagnetic records from CBI and KNY. Most of the simultaneous variations in these records are explained by a weak magnetic storm on 11 March 2011 (Japan Meteorological Agency 2013). However, the record of $Z$ at CBI showed anomalous behavior starting around 7:00 (UTC) that could not be explained by the external source (see Appendix 1).

\section{Comparison of tide gauge and magnetic records}

Before comparing the magnetic and tidal records from Chichijima Island, they were band-pass filtered with a period of 3-60 min to remove diurnal and long-term signals. The resulting waveforms of sea-level change and the magnetic $Z$ component were strikingly similar,

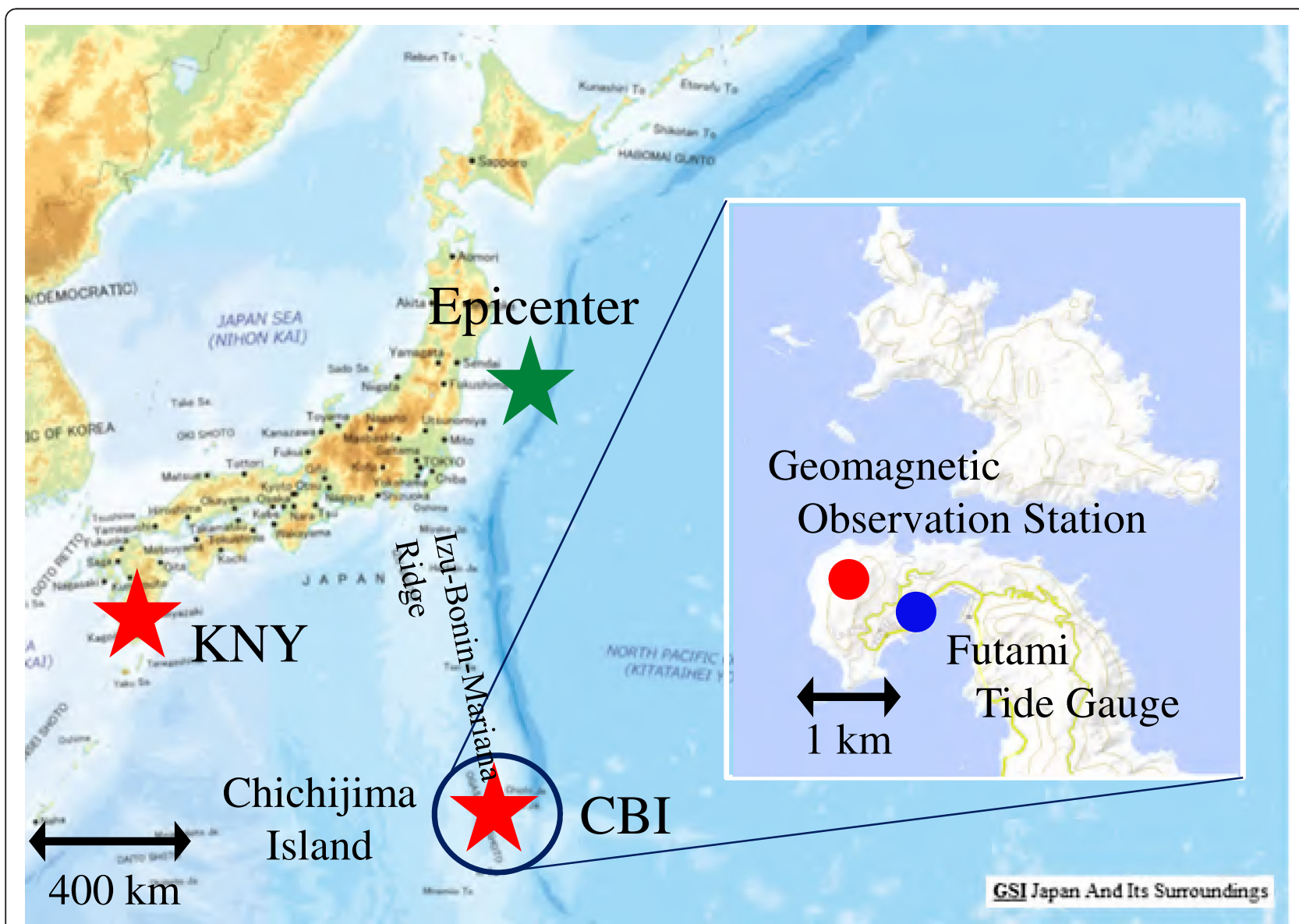

Fig. 1 Locations of geomagnetic stations CBI (inset: map of Chichijima Island with locations of the tide gauge station and geomagnetic observation station) and KNY as well as the epicenter of the 2011 Tohoku earthquake, as determined by JMA, are shown. Background image from the Geospatial Information Authority of Japan 

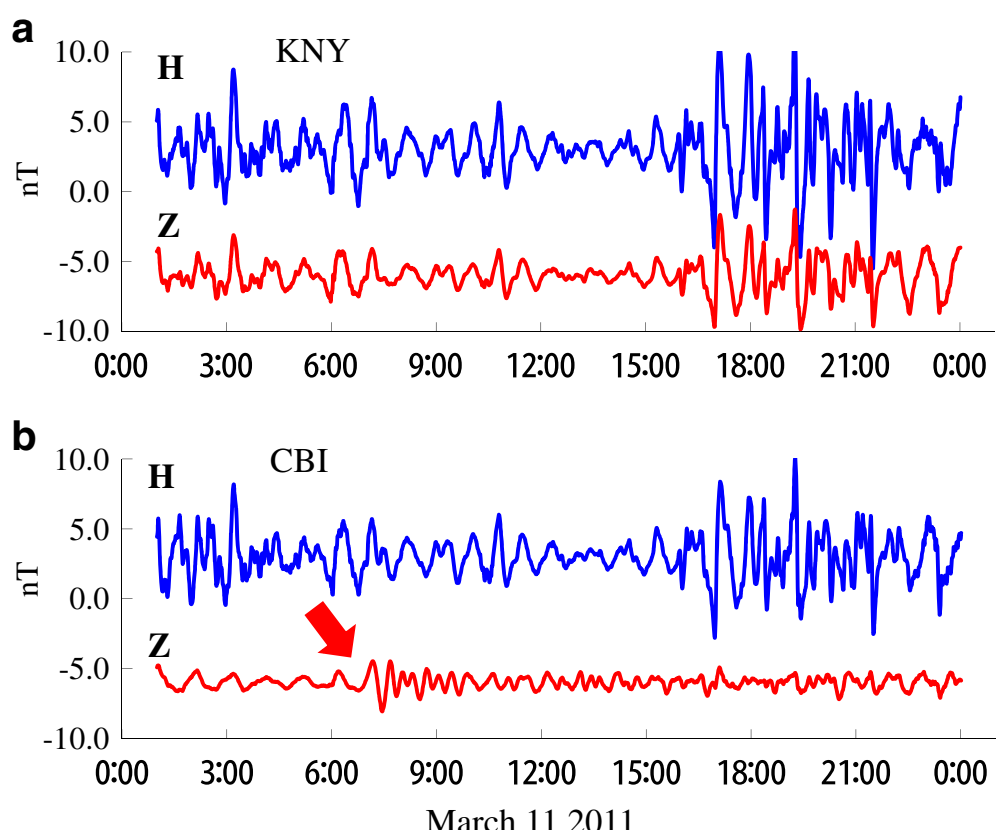

Fig. 2 Magnetic field observations at a Kanoya (KNY) and $\mathbf{b}$ Chichijima (CBI). The blue line is the horizontal component $H$ and the red line is the vertical component $Z$. The red arrow indicates the arrival of the anomalous signal associated with the tsunami

displaying seven sharp peaks between 7:00 and 9:30 (UTC) (Fig. 3).

Tidal records show that the tsunami arrived at 07:11 (UTC), with a maximum height (half amplitude) of $182 \mathrm{~cm}$ (Japan Meteorological Agency 2013). The dominant period of the tsunami between the first and seventh waves was around $20 \mathrm{~min}$. The maximum amplitude of $Z$, observed in the second peak, was $1.6 \mathrm{nT}$. The predominant period of both waveforms was consistently about $20 \mathrm{~min}$. The arrival times of the magnetic

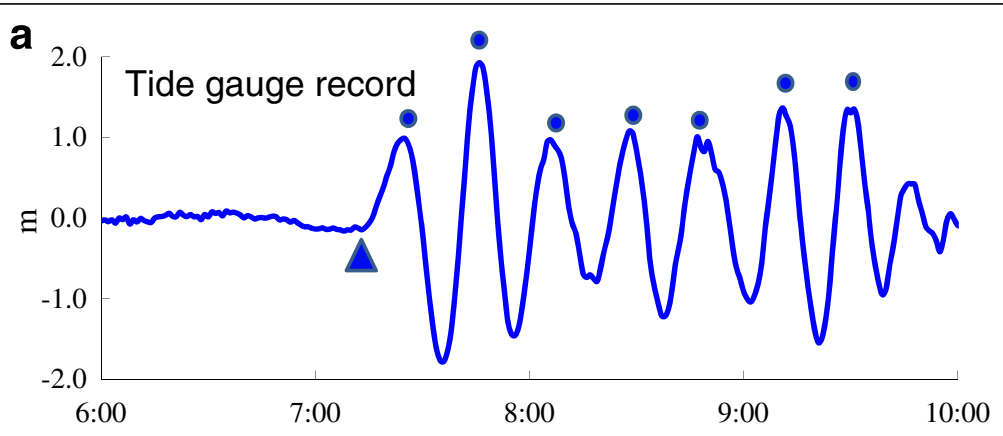

b

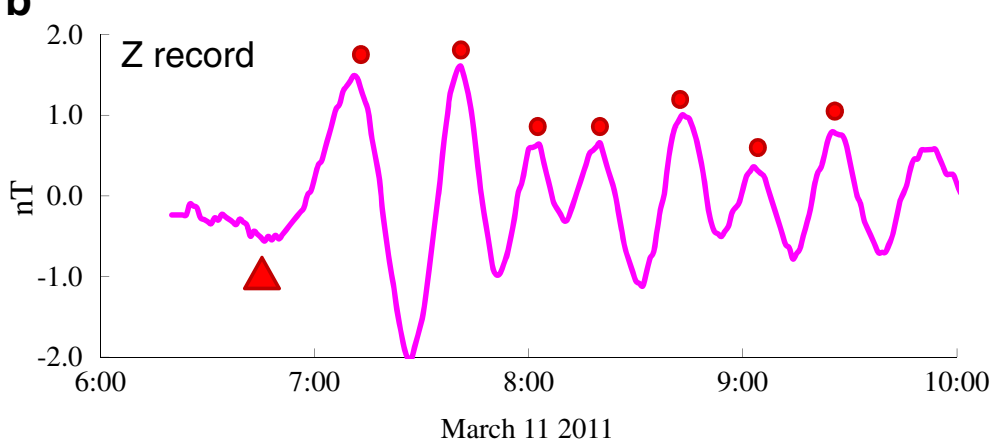

Fig. 3 a Tide gauge record and $\mathbf{b}$ Z record observed at Chichijima Island on 11 March 2011 (UTC). Triangles indicate the arrivals of the tsunami and the magnetic signal; peaks are indicated by small circles 
signal were recorded at 06:50 showing that the initial magnetic signal preceded the initial tide gauge signal by about $20 \mathrm{~min}$. The first peak of the magnetic signal preceded that of the tsunami wave by about $14 \mathrm{~min}$, and the third through seventh magnetic peaks preceded those of the tsunami waves by about $5 \mathrm{~min}$. For our purposes, the locations of the tidal and magnetic stations can be considered the same. Thus, the magnetic variations were detected 5-20 min ahead of the tsunami.

\section{Simulations of tsunami flows and induced magnetic fields} We performed a numerical tsunami simulation that explains the tsunami observations at Chichijima Island and nearby offshore tide gauges. Then we computed the magnetic variation induced by the oceanic dynamo effect and compared the results to the magnetic observations. Our analysis involved three steps: first, computation of the flow of seawater in the simulation; second, computation of the electric current induced by seawater flow; and third, computation of the resulting magnetic field variations on the basis of the Biot-Savart law.

\section{Tsunami flow model}

Zhang et al. (2014b) studied the induced magnetic field at CBI through a numerical simulation. The amplitude of their computed magnetic waveform was slightly higher than that of the observation. They mentioned that the reason might be the low accuracy of the numerical tsunami simulation, which used a planar coordinate system.

We used a nonlinear numerical tsunami model that takes seabed friction and Coriolis force into consideration, where one grid size is $1 \mathrm{~min}$ of arc (along longitude and latitude), the time difference is $3.06 \mathrm{~s}$, and the system of coordinates is spherical.
The computed and observed tsunami waveforms are shown in Fig. 4. Details of the tsunami model, earthquake fault parameters, and incorporation of data from buoys of Deep-ocean Assessment and Reporting of Tsunamis (DART) are presented in Appendix 2.

The computed waveform was sufficiently accurate considering the tsunami flow around Chichijima Island.

\section{Induced electric current and magnetic field}

The induction of electric currents from tsunami waves is depicted schematically in Fig. 5a. We computed the induced electric currents using the previously computed seawater flow. Ichihara et al. (2013) provided the theoretical details behind this computation.

Tsunami waves could be approximated with sufficient accuracy by plane waves because the tsunami model described in the previous section used a small grid size of about $2 \times 2 \mathrm{~km}$. The electrical conductivity of seawater is assumed to be homogeneous in the vertical direction, and the electrical conductivities of the atmosphere and the seafloor are approximated at zero. The amplitude and phase shift of the induced current $J$ are then derived using the following expression:

$$
\mu J_{\perp}=-\frac{2 i}{1-i C_{d} / C} \frac{\eta F_{z}}{h}
$$

where $\mu$ is the magnetic permeability, $J_{\perp}$ is the total electric current density in the direction perpendicular to the propagation of the tsunami, $i$ is the imaginary unit, $C_{d}$ is the magnetic diffusivity (the phase velocity of the electric current wave in seawater), $C$ is the tsunami velocity, $\eta$ is the tsunami height, $F_{z}$ is the $Z$ component of the geomagnetic field $F$, and $h$ is the water depth. The imaginary unit represents the phase difference between the tsunami wave and the electric current in complex phase space. Details are presented in Appendix 3.

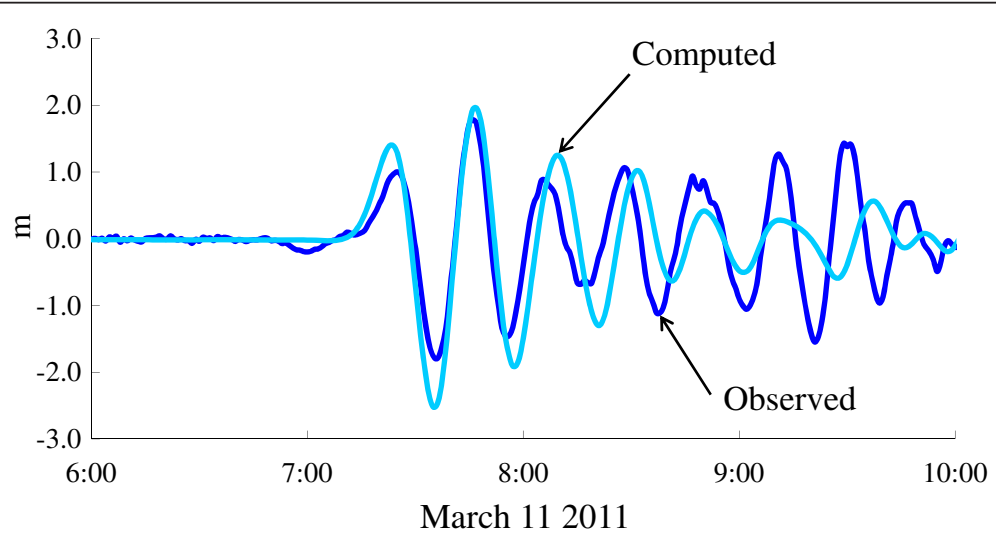

Fig. 4 Band-pass filtered tide gauge record from Chichijima Island showing observed tsunami waveforms (dark blue) and computed tsunami waveforms (light blue) 


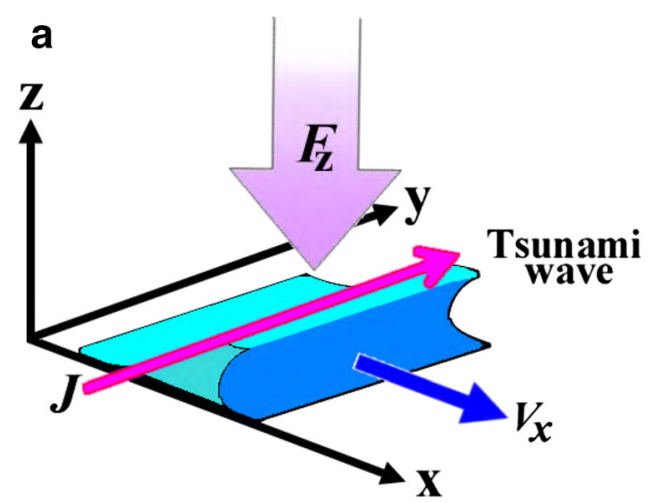

b

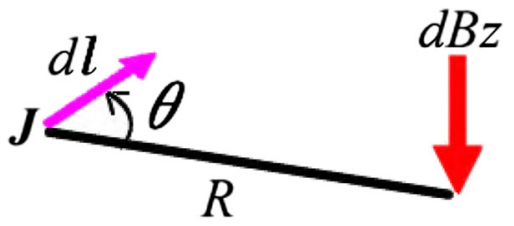

Fig. 5 Generation of induction of electric current by a tsunami according to the Biot-Savart law. a Relationship of geomagnetism, tsunami flow, and induced electric current, where $F_{z}$ is the $Z$ component of Earth's magnetism, $J$ is electric current density, and $V_{x}$ is flow velocity. b The Biot-Savart law shows the relation between $J$ and the induced magnetic field $d B_{Z \prime}$ expressed as $B_{Z}=\frac{\mu}{4 \pi} \frac{J d l \text { sin } \theta}{R^{2}}$, where $\mu$ is the magnetic permeability (see Appendix 3), $R$ is the distance between electric current and observation point, $d l$ is the micro distance, and $\theta$ is the angle between $d l$ and $R$

The specific computation steps are given below.

1. Time domain data of tsunami height for every numerical grid point are decomposed to frequency domain data by fast Fourier transform (FFT). The formula (1) converts the frequency domain data to electric currents in complex space. Inverse FFT is then used to convert the frequency domain data of the current to time domain data (in real space), expressed as the length of a current vector.

2. The flow direction of the tsunami is computed from the numerical tsunami model. According to Ampere's law, the electric current direction is obtained by rotated flow direction by $90^{\circ}$. The electric current density and its direction are then obtained for each grid cell.

3. The induced magnetic field at the observation point is computed from the induced current source in each grid cell according to the Biot-Savart law (Fig. 5b). The induced magnetic field at CBI is obtained by integrating a sufficient area centered on Chichijima Island.

The computed and observed magnetic field variations at CBI are shown in Fig. 6. From the first motion to the fourth peak, the computed magnetic field agreed well with the observations. For the fifth and the seventh peaks, the amplitude of the computed waveform was reduced, but the timing of the peaks and troughs was consistent.

In sum, our computed sea-level changes and magnetic field variations agreed well with the observations, although we used only a simple electrical conductivity structure. This result indicates that the tsunami-induced magnetic field variations at Chichijima Island could be suitably computed by assuming an insulating seafloor.

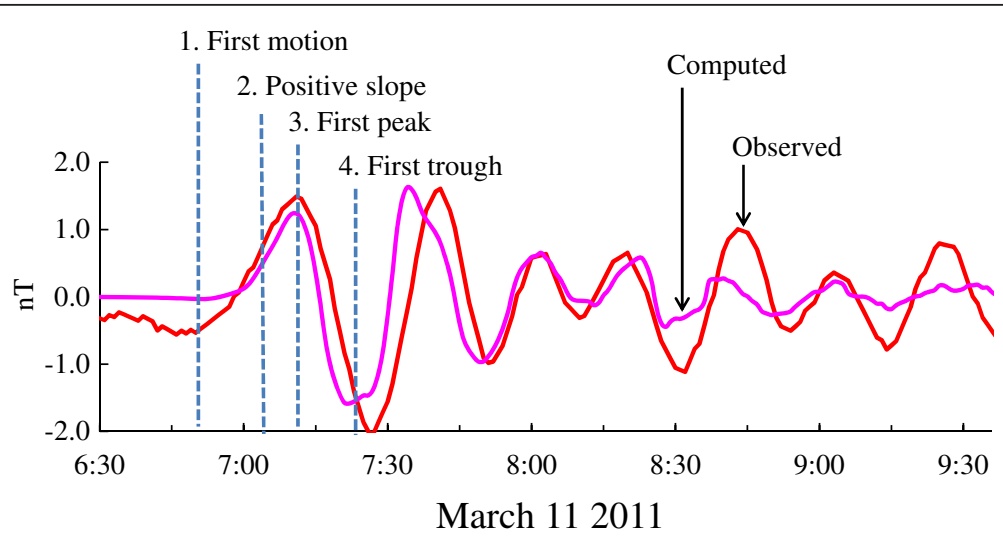

Fig. 6 Observed induced magnetic field waveform at CBI (red line) and computed waveform (purple line). Significant features of the waveform are labeled from one to four 


\section{Discussion}

To understand why the magnetic field variations preceded the sea-level changes at Chichijima Island, we compared the computed magnetic field variation to the patterns of seawater flow and induced electric currents around Chichijima Island (Fig. 7). Each distinctive phase from the first motion (6:53 UTC), the positive slope (7:05 UTC), the first peak (7:10 UTC),
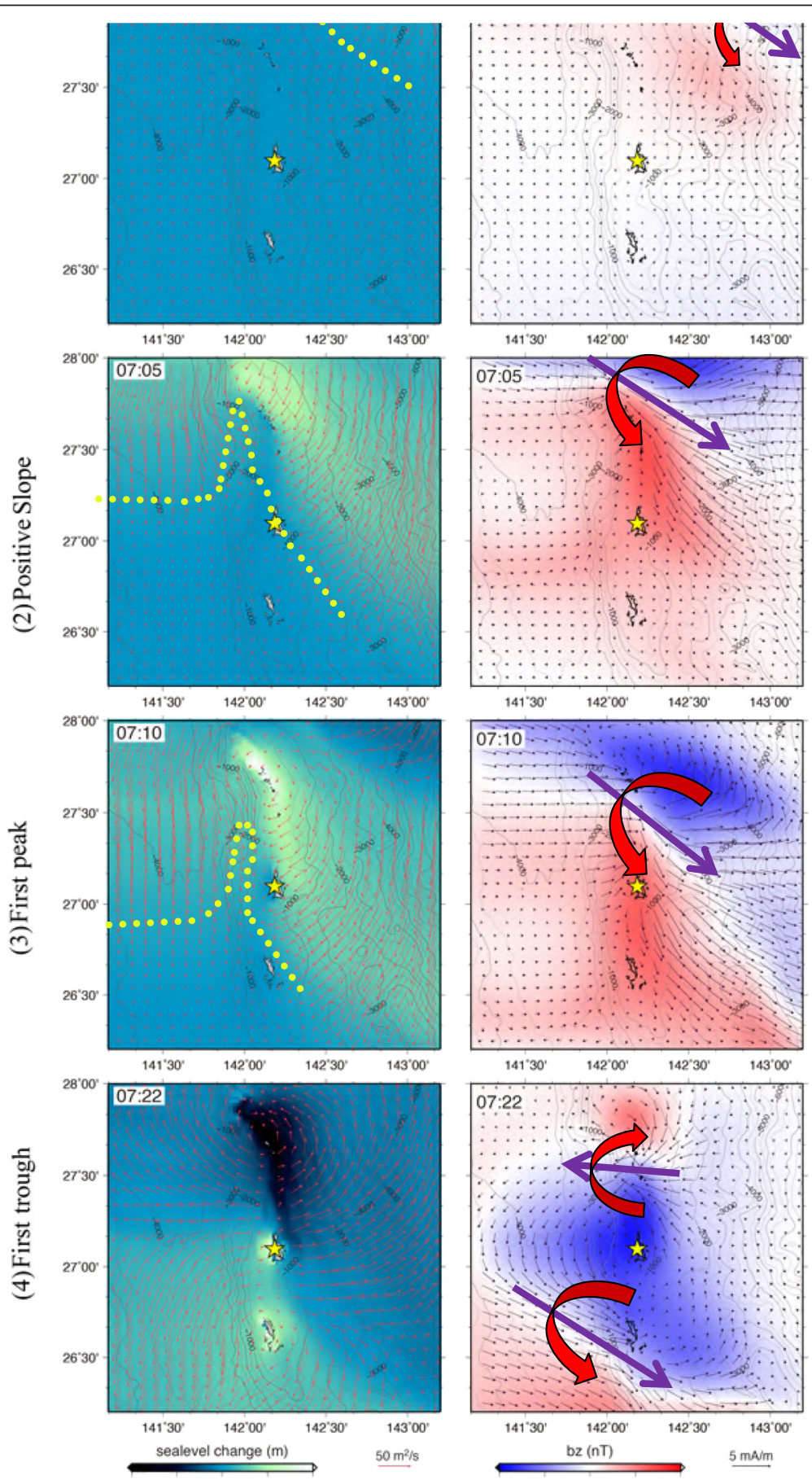

Fig. 7 Simulated tsunami wave height and velocity, and induced electromagnetic field around Chichijima Island (star). The left side shows wave height and seawater flow (depth-integrated velocity) as color images and vectors, respectively. The yellow dots indicate the tsunami fronts. The right side shows tsunami-induced magnetic and electric fields as color images and vectors, respectively. The labels on the left side indicate the phases of magnetic field variations. The large arrows indicate representative induced electric currents (purple vectors) and secondary induced magnetic fields (red arcs) 
and the first trough $(7: 22$ UTC) is explained as follows.

\section{First motion}

The flow of the first tsunami wave was more than $100 \mathrm{~km}$ northeast of Chichijima Island.

Simultaneously, a weak induced magnetic field extended to Chichijima Island.

2 Positive slope

The first tsunami wave was northeast of Chichijima Island. Positive and negative variations of the magnetic field were south (front) and farther north (back) of the tsunami wave, respectively.

3 First peak

The first tsunami wave was near the east coast of Chichijima Island. Induced electric currents near the island flowed from the north to the east. A positive variation of the magnetic field lay over the island, and the following negative variation moved south into the area shown in Fig. 7.

4 First trough

The first trough of the tsunami reached the northern side of Chichijima Island, and rip flows of the tsunami moved away from the island. A negative variation of the magnetic field lay over the island, and a new positive variation entered the area shown in Fig. 7, moving south.

Equation 14 indicates that an induced electric current is delayed when a tsunami wave is in a phase with the positive slope, but the phase-delay effect of the $C_{\mathrm{d}} / C$ term becomes weak as ocean depth decreases. As such, the induced electric current near the coastline is substantially in phase with the tsunami. Minami et al. (2015) discussed these phenomena in detail.

The first tsunami wave approaching Chichijima Island from the northeast induced an electric current in the leftward direction relative to the tsunami propagation. Around 7:05-7:10, northeast of Chichijima Island, there were large and parallel electric currents (purple arrows in Fig. 7). According to Ampere's right-hand rule, these currents induced large magnetic fields (red arcs in Fig. 7).

From this exercise, we conclude the following: if a tsunami wave is approximately planar, the lines representing the induced electric current and the peak of the tsunami wave are parallel and coincident, and thus, the induced magnetic field must curl over both the electric current and the tsunami wave. Because this induced magnetic field can reach beyond the tsunami wave, its signal precedes the wave and can be detected at least several minutes before the tsunami's arrival.

The amplitudes of the induced magnetic field and the tsunami's height are related with an approximate ratio of
$1 \mathrm{nT} / \mathrm{m}$. Four other examples of induced magnetic phenomena recorded at CBI had similar ratios (Tatehata 2015). This sensitivity is sufficient for detecting tsunamis with height of above $1 \mathrm{~m}$, considering the typical signalto-noise ratio of geomagnetic observations. Our findings suggest the possibility that magnetic field observations can serve as a method for rapid detection of imminent tsunamis.

\section{Conclusion}

We showed that the vertical component of the geomagnetic field and the tide gauge records at Chichijima Island exhibited very similar patterns as the tsunami caused by the 2011 Tohoku earthquake. The first magnetic signal preceded the arrival of the first tsunami wave at the Chichijima Island tide gauge by about $20 \mathrm{~min}$.

We quantitatively modeled the tsunami and the corresponding magnetic field variations at Chichijima Island. Not only was the computed magnetic field signal in close agreement with the observed signal, but also the mechanism before the arrival of the tsunami wave was quantitatively explained. Our numerical simulation showed that the straight-line electric current induced a secondary magnetic field that curled at the front of the first tsunami wave.

\section{Appendix 1}

\section{Effect of external geomagnetic disturbance}

Due to external sources, the horizontal component $H$ of the geomagnetic field induces the vertical component $Z$ depending on the lateral resistivity gradient in the subsurface. The transfer function of $\mathbf{T}$ between horizontal $H$ and vertical $Z$ magnetic components is

$$
Z=T H
$$

where $\mathbf{T}$ is a complex number. The amplitude of $\mathbf{T}$ at stations KNY and CBI estimated from Eq. 2 is $>0.40$ and $<0.15$, respectively, in the period band between 1000 and 10,000 s (Additional file 1: Figure S1). This indicates that $Z$ induced during geomagnetic storms should be relatively large at KNY, but relatively small at CBI.

Sounding curves of geomagnetic transfer functions $(\mathbf{T})$ at stations CBI and KNY determined using the BIRRP program (Chave and Thomson 2004).

The long-period soundings (320-61,440 s) were estimated from geomagnetic records between 1 April and 30 July 2010. We used the remote reference technique (Gamble et al. 1979) with the data from CBI and KNY for the long-period sounding of KNY and CBI, respectively. The short-period soundings (5.33-256 s) were estimated from the records between 8 and 10 March 2011. 
A weak geomagnetic storm was observed on 11 March 2011 (Japan Meteorological Agency 2013), and significant variations of $H$ were observed at $\mathrm{CBI}$ and KNY (Fig. 3). The strong correlation between $Z$ and $H$ indicates that an external geomagnetic disturbance induced $Z$ at KNY, as the relatively high value of $\mathbf{T}$ implies. On the other hand, the small value of $\mathbf{T}$ for CBI does not explain the amplitude of $Z$ between 7:00 and 10:00 (UTC). In addition, $H$ and $Z$ did not appear to be correlated during this period.

To evaluate the effect of the external disturbance on $Z$ at CBI quantitatively, we computed $Z$ from the estimated $\mathbf{T}$ and the observed $H$ using Eq. 2. The computed $Z$ waveform closely matched the observed data until 7:00, when large oscillations were observed (Additional file 2: Figure S2). Thus, secondary geomagnetic variations of external origin accounted for the observations before 7:00 but not after 7:00.

\section{Appendix 2}

\section{Tsunami simulation}

To simulate the tsunami around Chichijima Island, we used the following numerical tsunami model, which uses the long-wave approximation and takes into account the shallow bottom friction:

$$
\begin{aligned}
\frac{\partial Q_{\phi}}{\partial \mathrm{t}}= & -g h \frac{1}{R \cos \lambda} \frac{\partial \eta}{\partial \phi}-\frac{1}{h}\left\{Q_{\phi} \frac{1}{R \cos \lambda} \frac{\partial Q_{\phi}}{\partial \phi}+Q_{\lambda} \frac{\partial Q_{\phi}}{\partial \lambda}\right\} \\
& -\gamma \frac{1}{h^{2}} Q_{\phi} \sqrt{Q_{\phi}{ }^{2}+Q_{\lambda}{ }^{2}}-f Q_{\lambda} \\
\frac{\partial Q_{\lambda}}{\partial \mathrm{t}}= & -g h \frac{1}{R \cos \lambda} \frac{\partial \eta}{\partial \phi}-\frac{1}{h}\left\{Q_{\phi} \frac{1}{R \cos \lambda} \frac{\partial Q_{\lambda}}{\partial \phi}+Q_{\lambda} \frac{\partial Q_{\lambda}}{\partial \lambda}\right\} \\
& -\gamma \frac{1}{h^{2}} Q_{\lambda} \sqrt{Q_{\phi}{ }^{2}+Q_{\lambda}{ }^{2}}+f Q_{\phi} \\
\frac{\partial \eta}{\partial \mathrm{t}}= & -\left\{\frac{1}{R \cos \lambda} \frac{\partial Q_{\phi}}{\partial \phi}+\frac{\partial Q_{\lambda}}{\partial \mathrm{y}}\right\},
\end{aligned}
$$

where $R$ is the Earth's radius, $\lambda$ is the latitude, $\phi$ is the longitude, $h$ is the water depth, $g$ is the gravity, $\gamma$ is the friction coefficient of the seabed, $f$ is the Coriolis coefficient, and $\eta$ is the uplift of the sea surface. $Q_{\phi}$ and $Q_{\lambda}$ denote vertically integrated flow rates, which are used for the computation of induced electric currents. In this simulation, we used a 1-arcmin-resolution numerical model and the bathymetry dataset ETOPO1 (National Geophysical Data Center).

In numerical simulations of tsunamis, fault models with multiple small active segments are used to produce the initial tsunami wave. For example, Maeda et al. (2011) used 12 small faults, and Satake et al. (2013) used 55 small faults. However, we devised a simple fault model for the purpose described in the main text. In accordance with the Chichijima tide record and DART buoys located south of the island, arrival times, amplitude, period, and the shape of the tsunami wave were simulated. Finally, we obtained a single simple fault model, centered at $37.88^{\circ} \mathrm{N}, 143.23^{\circ} \mathrm{E}$, with the following parameters: strike $203^{\circ}$, length $186 \mathrm{~km}$, width $129 \mathrm{~km}$, slip $25 \mathrm{~m}$, dip angle $16^{\circ}$, slip angle $101^{\circ}$, depth $5.1 \mathrm{~km}$, and slip time function $180 \mathrm{~s}$. The initial uplift of the sea surface from this fault model is shown in Additional file 3: Figure S3.

The resulting computed and observed tsunami waveforms at six DART buoys in the western Pacific Ocean are shown in Additional file 4: Figure S4. The computed waveforms at the Chichijima tide station (Fig. 4), DART 52403, and DART 52405 in the "South Area" (Additional file 4: Figure S4) show good agreement with the observations. Hence, this computation has sufficient accuracy to model the tsunami flow $\left(Q_{\phi}, Q_{\lambda}\right)$ passing through the Chichijima Island area.

\section{Appendix 3}

\section{Derivation of the formulae}

The induced magnetic field equation of a conductor is represented as

$$
\frac{\partial \boldsymbol{B}}{\partial t}=\nabla \times(\boldsymbol{u} \times \boldsymbol{B})-\nabla \times(K \nabla \times \boldsymbol{B})
$$

where $K$ is the magnetic diffusivity, $u$ is the flow velocity vector of seawater, and $B$ is the magnetic field. The magnetic field $B$ can be decomposed to $B=F+b$, where $F$ is the geomagnetic field and $b$ is the induced magnetic field.

In a homogeneous conductor, the induction equation for the vertical component $b_{\mathrm{z}}$ is

$$
\frac{\partial b_{z}}{\partial t}=-\nabla_{H} \cdot\left(F_{z} \boldsymbol{u}_{\boldsymbol{H}}\right)+K \nabla^{2} b_{z}
$$

The derivation of Eq. 5 is detailed in Tyler (2005) and Ichihara et al. (2013).

The general solution for Eq. 5 is expressed in the following form, in which the coefficient constants $D_{1}, D_{2}$ are determined by boundary conditions:

$$
b_{z}=-\frac{1}{2 \alpha^{2} K} \nabla_{H} \cdot\left(F_{Z} \boldsymbol{u}_{H}\right)\left[D_{1} e^{\alpha x}+D_{2} e^{-\alpha x}+2\right]
$$

where $\alpha:=\sqrt{\kappa^{2}-i \omega / K}, \omega$ is the frequency, $\kappa$ is the wavenumber vector describing the horizontal variation, and $x$ is the position vector, signifying a uniform plane wave.

From Maxwell's equations, the horizontal component $b_{H}$ of the tsunami propagation direction is expressed as 


$$
\begin{aligned}
b_{H} & =-\frac{1}{i \kappa} \frac{\partial b_{z}}{\partial z} \\
& =-\frac{i}{2 \alpha \kappa K} \nabla_{H} \cdot\left(F_{z} \boldsymbol{u}_{H}\right)\left[D_{1} e^{\alpha z}-D_{2} e^{-\alpha z}\right] .
\end{aligned}
$$

The boundary conditions for seawater are that $b_{\mathrm{z}}$ and $\partial b_{\mathrm{z}} / \partial \mathrm{z}$ are continuous at the upper (sea surface) and lower (seabed) boundaries. The latter condition means that the horizontal component of the magnetic field is continuous at the boundary.

For the coordinate system, the $z$-direction is perpendicular to the sea surface, $z=0$ is the sea surface, and $z=-h$ is the seabed ( $h$ is water the depth). The $x$-direction is to the east and the $y$-direction is to the north. In the air above the sea surface and the crust below the seabed, $\nabla^{2} b_{\mathrm{z}}=0$.

Then the boundary condition Eq. 7 is reduced as follows:

$$
\frac{\partial^{2}}{\partial z^{2}} b_{z}-\kappa^{2} b_{z}=0 .
$$

In addition, there are other boundary conditions for which the solutions converge to zero at infinite distances from the seawater:

$$
b_{z}=P e^{-\kappa z} \quad(0 \leq z), b_{z}=Q e^{\kappa(z+h)} \quad(z \leq-h) .
$$

From Eqs. 6 and 7 and the boundary conditions, the coefficients $D_{1}, D_{2}, P$, and $Q$ are obtained as

$$
\begin{aligned}
& D_{1}=-\frac{\kappa e^{\frac{\alpha h}{2}}}{\kappa \cosh \frac{\alpha h}{2}+\alpha \sinh \frac{\alpha h}{2}}, D_{2}=-\frac{\kappa e^{\frac{-\alpha h}{2}}}{\kappa \cosh \frac{\alpha h}{2}+\alpha \sinh \frac{\alpha h}{2}}, \\
& P=\frac{-1}{\alpha K} \nabla_{H} \cdot\left(F_{z} \boldsymbol{u}_{H}\right) \frac{\sinh \frac{\alpha h}{2}}{\kappa \cosh \frac{\alpha h}{2}+\alpha \sinh \frac{\alpha h}{2}}, \\
& Q=\frac{-1}{\alpha K} \nabla_{H} \cdot\left(F_{z} \boldsymbol{u}_{H}\right) \frac{\sinh \frac{\alpha h}{2}}{\kappa \cosh \frac{\alpha h}{2}+\alpha \sinh \frac{\alpha h}{2}} .
\end{aligned}
$$

Therefore, Eq. 6 can be rewritten as

$$
b_{Z}=\frac{-1}{\alpha^{2} K} \nabla_{H} \cdot\left(F_{z} \boldsymbol{u}_{H}\right)\left[1-\frac{\sinh \alpha\left(z+\frac{h}{2}\right)}{\kappa \cosh \frac{\alpha h}{2}+\alpha \sinh \frac{\alpha h}{2}}\right] \text {. }
$$

Equation 8 is differentiated to obtain

$$
\frac{\partial b_{Z}}{\partial z}=\frac{\kappa}{\alpha K} \nabla_{H} \cdot\left(F_{z} \boldsymbol{u}_{H}\right)\left[1-\frac{\sinh \alpha\left(z+\frac{h}{2}\right)}{\kappa \cosh \frac{\alpha h}{2}+\alpha \sinh \frac{\alpha h}{2}}\right] .
$$

Moreover, $b_{H}$ is obtained by rewriting Eq. 7:

$$
b_{H}=\frac{i}{\alpha K} \nabla_{H} \cdot\left(F_{z} \boldsymbol{u}_{H}\right) \frac{\sinh \alpha\left(z+\frac{h}{2}\right)}{\kappa \cosh \frac{\alpha h}{2}+\alpha \sinh \frac{\alpha h}{2}} .
$$

As the next step, we obtained the electric current in the seawater flow arising from the induced magnetic field $b$. Using the Maxwell equation for current density $j$, this electric current is determined by

$$
\mu \boldsymbol{j}=\nabla \times \boldsymbol{b}
$$

where $\mu$ is the magnetic permeability and $j$ is the vector of current density.

Assuming a plane wave solution with wavenumber $\kappa$, the magnetic field vector $b$ is decomposed as

$$
b_{x}=\frac{\kappa_{x}}{\kappa} b_{H}, \quad b_{y}=\frac{\kappa_{y}}{\kappa} b_{H} .
$$

Then, $\mu j$ is described as

$$
\begin{aligned}
& \mu j_{x}=i \kappa_{y} b_{z}-\frac{\kappa_{y}}{\kappa} \frac{\partial b_{H}}{\partial z}=-\frac{\kappa_{y}}{\kappa}\left(-i \kappa b_{z}+\frac{\partial b_{H}}{\partial z}\right), \\
& \mu j_{y}=i \kappa_{x} b_{z}-\frac{\kappa_{x}}{\kappa} \frac{\partial b_{H}}{\partial z}=-\frac{\kappa_{x}}{\kappa}\left(-i \kappa b_{z}+\frac{\partial b_{H}}{\partial z}\right), \\
& \mu j_{x}=i \kappa_{x} b_{y}-i \kappa_{y} b_{x}=i \kappa_{x} \frac{\kappa_{y}}{\kappa} b_{H}-i \kappa_{y} \frac{\kappa_{x}}{\kappa} b_{H}=0 .
\end{aligned}
$$

That is, the current density in the direction perpendicular to the propagation of the tsunami is

$$
\mu j_{\perp}=-i \kappa b_{\mathrm{z}}+\frac{\partial b_{H}}{\partial z}
$$

To compute the induced magnetic field at the Chichijima geomagnetic observatory, we require the total amount of current at density $J_{\perp}$ in seawater, which is obtained by integrating Eq. 11 from the seabed to the sea surface. Hence,

$$
\mu J_{\perp}=-i \kappa \int_{-h}^{0} b_{z} d z+\left.b_{H}\right|_{-h} ^{0}
$$

is the integral equation to be solved. Using Eqs. 6 and 7, the terms on the right side of Eq. 12 can be rewritten as

$$
\begin{aligned}
i \kappa \int_{-h}^{0} b_{z} d z= & \frac{i \kappa}{2 \alpha^{2} K} \nabla_{H} \cdot\left(F_{z} u_{H}\right)\left\{2 h+\frac{1}{\alpha}\left(D_{1}-D_{1}\right)\right. \\
& \left.-\frac{1}{\alpha}\left(D_{1} e^{-\alpha h}-D_{1} e^{\alpha h}\right)\right\}
\end{aligned}
$$

and

$$
\begin{aligned}
& b_{H}(z=0)=-\frac{i}{2 \alpha \kappa K} \nabla_{H} \cdot\left(F_{z} \boldsymbol{u}_{H}\right)\left[D_{1}-D_{2}\right], \\
& b_{H}(z=-h)=-\frac{i}{2 \alpha K K} \nabla_{H} \cdot\left(F_{z} \boldsymbol{u}_{H}\right)\left[D_{1} e^{-\alpha h}-D_{2} e^{\alpha h}\right] .
\end{aligned}
$$

Therefore, the total electric current is 


$$
\begin{aligned}
\mu J_{\perp}= & -\frac{i}{\alpha^{2} K} \nabla_{H} \cdot\left(F_{z} \boldsymbol{u}_{H}\right) \\
& \left\{\kappa h+2\left(1-\frac{\kappa^{2}}{\alpha^{2}}\right) \frac{\sinh \alpha h+\frac{\kappa}{\alpha}(\cosh \alpha h-1)}{\left\{\left(\frac{\kappa}{\alpha}\right)^{2}+1\right\} \sinh \alpha h+2 \frac{\kappa}{\alpha} \cosh \alpha h}\right\} .
\end{aligned}
$$

Here, we use the continuity equation of the tsunami and the long-wave approximation $\left(u_{\mathrm{z}} \ll\left|u_{\mathrm{H}}\right|\right)$ to simplify the flow rate divergence equation by using the height $\eta$ of the tsunami:

$$
\begin{aligned}
& \frac{\partial \eta}{\partial t}+\nabla \cdot\left(h \boldsymbol{u}_{H}\right)=0 \\
& \nabla_{H} \cdot\left(F_{z} \boldsymbol{u}_{H}\right)=\frac{i \omega \eta F_{z}}{h}
\end{aligned}
$$

Using the approximation $h \ll \lambda$, Eq. 13 can be rewritten as

$$
\mu J_{\perp}=-\frac{\omega}{\alpha^{2} K} \frac{\eta F_{z}}{h}\left\{\frac{2 \sinh \alpha h}{\left\{\left(\frac{\kappa}{\alpha}\right)^{2}+1\right\} \sinh \alpha h+2 \frac{\kappa}{\alpha} \cosh \alpha h}\right\} .
$$

Using skin depth $(\delta:=\sqrt{2 K / \omega}))$ and the approximation $\delta \ll \lambda, \mu J_{\perp}$ can be further simplified:

$$
\mu J_{\perp} \approx \frac{-\omega}{K} \frac{\eta F_{z}}{h}\left\{\frac{2}{-2 i \frac{1}{\delta^{2}}+2 \frac{k}{h}}\right\}=-\frac{\eta F_{\mathrm{z}}}{h}\left\{\frac{2}{-i+\frac{2 K}{h C}}\right\}
$$

Therefore,

$$
\mu J_{\perp}=-\frac{2 i}{1-i C_{d} / C} \frac{\eta F_{z}}{h}
$$

is obtained, where $C_{d}:=2 K / h$ and $C:=\sqrt{g h}$. This formulation uses only the approximations $h \ll \lambda$ and $\delta \ll \lambda$, and does not use $h \ll \delta$. Hence, Eq. 14 can be applied to tsunamis deeper than the skin depth. The direction of the electric current is estimated by computing the flow velocity vectors.

This derivation assumed that the electrical conductivity of the seafloor is zero. Ichihara et al. (2013), Sugioka et al. (2014), and Minami et al. (2015) described a more extended formulation using a seafloor with finite electrical conductivity. Zhang et al. (2014b) considered the electrical conductivity of the seafloor as a threedimensional multi-layer structure. However, Tada et al. (2014) showed that the electrical conductivity of the seafloor is less than $0.1 \mathrm{~S} / \mathrm{m}$ around Chichijima Island. Hence, the conductivity of the sea floor can be approximated to zero.

\section{Additional files}

Additional file 1: Figure S1. Sounding curves of geomagnetic transfer functions ( $\mathbf{T}$ ) at stations $\mathrm{CBI}$ and $\mathrm{KNY}$ determined using the BIRRP program (Chave and Thomson 2004). (PDF $56 \mathrm{~kb}$ )

Additional file 2: Figure S2. Observed geomagnetic waveform and computed $Z$ at CBI. (GIF 15,148 kb)

Additional file 3: Figure S3. Results of the fault displacement model (initial tsunami wave) showing the vertical movement of the crust. (PDF $27 \mathrm{~kb}$ )

Additional file 4: Figure S4. Map showing tsunami propagation along with DART buoys (yellow squares) and their observed and computed sea-level waveforms (dark and light blue lines, respectively). Tsunami travel times were obtained from NOAA; the contour interval is $1 \mathrm{~h}$. The measurement for DART buoys (red squares; 5204 and 52401) was omitted. (PDF $421 \mathrm{~kb}$ )

\section{Competing interests}

The authors declare that they have no competing interests.

\section{Authors' contributions}

HT carried out the numerical simulations: Tsunami and induced Magnetic field and was responsible the draft of the manuscript. HI performed the evaluation of the magnetic signal and revised the first draft to submission stage. $\mathrm{YH}$ discovered the magnetic signal from the Tsunami at $\mathrm{CBI}$, formulated the electric current and tsunami flow theoretically, and coordinated in this study. All authors read and approved the final manuscript.

\section{Acknowledgements}

We thank Kenji Satake for the fruitful discussions regarding the highresolution fault model and the reviewers for helping us substantially improve this paper. We thank the JMA Global Environment and Marine Department for the tidal data, the Kakioka Magnetic Observatory for the magnetic field data at Chichijima Island, and the U.S. National Oceanic and Atmospheric Administration for the DART buoy data, which are available at https:// www.ngdc.noaa.gov/hazard/dart/2011honshu_dart.html. We dedicate this paper to the 23,000 victims of the Tohoku earthquake tsunami.

\section{Author details}

${ }^{1}$ Kakioka Magnetic Observatory, Japan Meteorological Agency, 595 Kakioka, Ishioka-shi, Ibaraki-ken 315-0116, Japan. ²Research and Development Center for Earthquake and Tsunami, Japan Agency for Marine-Earth Science and Technology, 2-15 Natsushima-cho, Yokosuka 237-0061, Japan. ${ }^{3}$ Graduate School of Maritime Sciences, Kobe University, 5-1-1 Fukae-minamimachi, Higashinada-ku, Kobe 658-0022, Japan. ${ }^{4}$ Department of Deep Earth Structure and Dynamics Research, Japan Agency for Marine-Earth Science and Technology, 2-15 Natsushima-cho, Yokosuka 237-0061, Japan.

Received: 19 April 2015 Accepted: 23 October 2015

Published online: 19 November 2015

\section{References}

Chave AD, Thomson DJ (2004) Bounded influence magnetotelluric response function estimation. Geophys J Int 157:988-1006. doi:10.1111/J.1365246x.2004.02203.X

Faraday M (1832) The Bakerian lecture: experimental researches in electricity—second series. Philos Trans R Soc London 122:163-194. doi:10.1098/rstl.1832.0007

Gamble TD, Clarke J, Goubau WM (1979) Magnetotellurics with a remote magnetic reference. Geophysics 44:53-68

Ichihara H, Hamano Y, Baba K, Kasaya T (2013) Tsunami source of the 2011 Tohoku earthquake detected by an ocean-bottom magnetometer. Earth Planet Sc Lett 382:117-124

Japan Meteorological Agency (2013) Report of the Kakioka Magnetic Observatory 2011, CD-ROM

Maeda T, Furumura T, Sakai S, Shinohara M (2011) Significant tsunami observed at ocean-bottom pressure gauges during the 2011 Off the Pacific Coast of Tohoku earthquake. Earth Planets Space 63(7):803-808 
Manoj C (2011) Observation of magnetic fields generated by tsunamis. Eos 92:13-14

Minami T, Toh H, Tyler H (2015) Properties of electromagnetic fields generated by tsunami first arrivals: classification based on the ocean depth. Geophys Res Let. doi:10.1002/2015GL063005

Sanford TB (1971) Motionally induced electric and magnetic fields in the sea. J Geophys Res 76:3476-3492. doi:10.1029/JC076i015p03476

Satake K, Fujii F, Harada T, Namegaya Y (2013) Time and space distribution of coseismic slip of the 2011 Tohoku Earthquake as inferred from tsunami waveform data. Bul Seism Soc Am 103(2B):1473-1492. doi:10.1785/ 0120120122,2013

Sugioka H, Hamano Y, Baba K, Kasaya T, Tada N, Suetsugu D (2014) Tsunami: ocean dynamo generator. Scientific Reports 4:3596. doi:10.1038/srep03596

Tada N, Baba K, Utada H (2014) Three-dimensional inversion of seafloor magnetotelluric data collected in the Philippine Sea and the western margin of the northwest Pacific Ocean. Geochem Geophys Geosyst 15:2895-2917. doi:10.1002/2014GC005421

Tatehata H (2015) Tsunami-induced magnetic field records at the Chichijima Island observation station. Tech Rep Kakioka Magnetic Obs 12(1-2):11-19 (in Japanese)

Toh H, Satake K, Hamano Y, Fujii Y, Goto T (2011) Tsunami signals from the 2006 and 2007 Kuril earthquakes detected at a seafloor geomagnetic observatory. J Geophys Res 116, B02104. doi:10.1029/2010JB007873

Tyler RH (2005) A simple formula for estimating the magnetic fields generated by tsunami flow. Geophys Res Let 32, L09608. doi:10.1029/2005GL022429

Tyler RH, Maus S, Luhr H (2003) Satellite observations of magnetic fields due to ocean tidal flow. Science 299:239-241. doi:10.1126/science.1078074

Zhang LL, Baba K, Liang PF, Shimizu H, Utada H (2014a) The 2011 Tohoku tsunami observed by an array of ocean bottom electro magnetometers. Geophys Res Lett 41(14):4937-4944. doi:10.1002/2014gl060850

Zhang LL, Utada H, Shimizu H, Baba K, Maeda T (2014b) Three-dimensional simulation of the electromagnetic fields induced by the 2011 Tohoku tsunami. J Geophys Res 119(1):150-168. doi:10.1002/2013jb010264

\section{Submit your manuscript to a SpringerOpen ${ }^{\circ}$} journal and benefit from:

- Convenient online submission

- Rigorous peer review

- Immediate publication on acceptance

- Open access: articles freely available online

- High visibility within the field

- Retaining the copyright to your article 